\title{
Nanofibers promote HepG2 aggregate formation and cellular function
}

\author{
W.T. Su${ }^{1}$, Y.J. Liu ${ }^{1}$ and T.Y. Huang ${ }^{2}$ \\ ${ }^{1}$ Department of Chemical Engineering and Biotechnology, \\ National Taipei University of Technology, Taipei, Taiwan \\ ${ }^{2}$ Department of Orthopedic Surgery Mackay Memorial Hospital, \\ Taipei, Taiwan \\ Corresponding author: W.T. Su \\ E-mail: f10549@ntut.edu.tw
}

Genet. Mol. Res. 15 (3): gmr.15038993

Received July 21, 2016

Accepted August 1, 2016

Published August 30, 2016

DOI http://dx.doi.org/10.4238/gmr.15038993

Copyright (C) 2016 The Authors. This is an open-access article distributed under the terms of the Creative Commons Attribution ShareAlike (CC BY-SA) 4.0 License

\begin{abstract}
Formation of hepatocyte spheroids is a necessary strategy for increasing liver-specific function in vitro. In this study, HepG2 cells showed good viability when grown on a polylactic acid-chitosan (PLACS) nanofiber and aggregated to form multicellular spheroids on the PLA-CS nanofibers with a diameter of approximately $100-200 \mathrm{~mm}$ in 5 days of culture, whereas no such aggregation was observed in cells cultured on 24-well plates. Hepatocyte spheroids formed on the PLACS nanofibers displayed excellent hepatic-related protein expression, such as albumin and urea, compared to HepG2 cells cultured on the 24-well plates. These results indicated that formation of the hepatocyte spheroids in nanofibers can increase and maintain hepatocyte functions for a longer time, supporting a new strategy for bioartificial liver development.
\end{abstract}

Key words: HepG2; Nanofiber; Albumin secretion; Urea synthesis 


\section{INTRODUCTION}

Tissue engineering for reconstructive organs requires an abundant cellular source, some suitable signals for cellular function, and a biocompatible and biodegradable scaffold for cellular attachment and proliferation. A bioartificial liver supports temporary liver function in vitro. However, when removed from the natural liver, hepatocytes rapidly lose their liverspecific function and viability. Cellular aggregation to spheroids has been shown to play an important role in supporting the viability, proliferation, and differentiation potential of hepatocytes in previous laboratory studies (Seo et al., 2006; Nakazawa et al., 2006; Chu et al., 2009). Therefore, formation of hepatocyte spheroids on scaffolds is a promising method to maintain long-term liver function in vitro.

Hepatocytes are anchorage cells; therefore, cells and substrate interactions can affect cellular aggregation (Su et al., 2015). The strength of cellular adhesion is a critical factor regulating the development of cellular clusters. The main characteristic of nanofibers is their ultrafine structure and that the fibers form continuous extensions to construct a threedimensional (3D) scaffold that possesses large porosity, uniform pore distribution, variable pore size, and a high surface-to-volume ratio, showing good potential to effectively mimic a natural environment for cellular growth. Electrospinning is used to transform the biopolymer solution into successive and thin fibers under a high electric field, facilitating the formation of a fibrous scaffold that can act as a substitute for natural extracellular matrices (ECM) to promote cellular growth (Su et al., 2015).

Polylactic acid (PLA) is a synthetic polyester that easily biodegrades in a natural environment, and has thus been widely used as a biomedical implant and biomaterial in tissue engineering. Chitosan (CS) is a polysaccharide composed of $\beta$-(1-4)-linked d-glucosamine and $\mathrm{N}$-acetyl-d-glucosamine. CS is found in many commercial and medical appliances with potential for use as an antibacterial agent and in drug delivery.

In this study, a PLA-CS blending solution was used to fabricate nanofibers for reducing the adherent area of cells and substrates to promote cellular aggregation in the formation of hepatocyte spheroids. The aim of this work was to develop a new strategy to increase and maintain the liver function of HepG2 cells in vitro.

\section{MATERIAL AND METHODS}

\section{PLA-CS nanofiber fabrication}

The PLA-CS fibrous scaffold was manufactured using an electrospinning system including an electrical power supply, syringe-pushed pump, Teflon ${ }^{\mathrm{TM}}$ tubing, a blunt $18-\mathrm{G}$ needle, and a collector. The high-voltage power was connected to the catheter tip, and aluminum foil was used as the collector owing to its electrical drag. PLA, CS, and trifluoroacetate were purchased from Sigma (St. Louis, MO, USA) and used without any further purification; 0.36 g CS and 0.18 g PLA were dissolved in $10 \mathrm{~mL}$ trifluoroacetate. The PLA-CS solution was poured into a $5-\mathrm{mL}$ syringe connected to an $18-\mathrm{G}$ needle at a $10-\mathrm{cm}$ tip-to-collector distance, with the flow rate set to $1.2 \mathrm{~mL} / \mathrm{min}$ by a pushed pump, and a polymer jet occurring under $25 \mathrm{kV}$. The fibers were continuously deposited on the aluminum foil to form a $3 \mathrm{D}$ nanofiber scaffold. The wettability of the PLA-CS fibrous scaffold was analyzed with a contact angle

Genetics and Molecular Research 15 (3): gmr.15038993 
meter (FACE, CA-D). Fourier transform infrared (FTIR) spectrometry (Horiba FT-720) of the PLA-CS nanofibers was guided in the mid-IR region between 800 and $4000 \mathrm{~cm}^{-1}$ at a 16scan process. Prior to spectroscopic measurements, all specimens were mixed with potassium bromide at a ratio of 5:95, and then all pellets were fixed in the sample holder.

\section{Cell culture and cell proliferation assay}

HepG2 is a human hepatocellular carcinoma-derived cell line that is a well-recognized model frequently used to investigate liver cell function in vitro. For this study, HepG2 cells were purchased from the Bioresource Collection and Resource Center (BCRC, Taiwan, China). The HepG2 cells were cultured in Dulbecco's modified Eagle medium containing $10 \%$ fetal bovine serum, and $1 \%$ penicillin and streptomycin, and incubated under $5 \% \mathrm{CO}_{2}$ at $37^{\circ} \mathrm{C}$. The culture medium was refreshed every two days. HepG2 cells were seeded in the fibrous scaffold and a 24-well plate (as a control) at a cellular density of $1 \times 10^{5}$ cells $/ \mathrm{mL}$. The cellular viability was measured using 3(4,5-dimethylthiazol-2-yl)-2,5-diphenyltetrazolium bromide (MTT) reagent. The proliferating cells were treated with $5 \mathrm{mg} / \mathrm{mL}$ MTT at $37^{\circ} \mathrm{C}$ after 2 days of culture. The culture medium was drawn out fully and the formed formazan product was dissolved in dimethyl sulfoxide. The metabolized crystals were measured according to the optical density in a spectrophotometer at $570 \mathrm{~nm}$ (Thermo Scientific Multiskan FC).

\section{Immunocytochemistry}

After 5 days of culture, HepG2 cells cultured on the PLA-CS nanofibers were rinsed twice with phosphate-buffered saline (PBS) to remove any unattached cells. To confirm the cellular aggregation, all cells were fixed in $4 \%$ formaldehyde for $10 \mathrm{~min}$ and then permeabilized on a cellular membrane containing $1 \%$ Triton X100. For immunocytochemistry, the cellular nuclei were stained with 4'6-diaminido-2-phenylindole, and filamentous actin was stained with phalloidin-tetramethylrhodamine B isothiocyanate. The immunostaining of all specimens was observed with a confocal laser-scanning microscope (Leica TCS-SP5-AOBS).

\section{Scanning electron microscopy (SEM) observation}

After 5 days of incubation, the HepG2 cells grown on the PLA-CS nanofibers were fixed in situ by $2.5 \%$ formaldehyde in PBS buffer at $4^{\circ} \mathrm{C}$ for $60 \mathrm{~min}$. Subsequently, all cells were rinsed with the PBS solution containing 5\% sucrose twice for $15 \mathrm{~min}$, dehydrated with alcohol solutions with a series of concentrations to $100 \mathrm{vol} \%$ ethanol, and then exposed to a $\mathrm{CO}_{2}$ critical point dryer (Pelco CPD\#2400) until completely dry. All dried specimens were covered with a thin layer of gold using a sputter coater (Cressington 108 manual), and then the cellular morphology was observed by SEM (FEI Quanta 200, Philips, the Netherlands).

\section{Functional evaluation by enzyme-linked immunosorbent assay (ELISA)}

To confirm hepatocyte function, the concentrations of albumin and urea in the medium were measured at different times of culture using a commercially available albumin assay kit (DIUR-250) and urea assay kit (DIUR-500) for ELISA according to the manufacturer

Genetics and Molecular Research 15 (3): gmr.15038993 
protocols. The secretion amount of albumin and the synthesis of urea were determined at 620 and $450 \mathrm{~nm}$, respectively, on an ELISA reader (Thermo Scientific Multiskan FC).

\section{Statistical analysis}

All experiments were performed at least in triplicate for different specimens. The results were assayed by analysis of variance and data are presented as means \pm standard deviation. Differences were considered to be statistically significant at $\mathrm{P}<0.05$.

\section{RESULTS AND DISCUSSION}

\section{Material characteristics of the PLA-CS nanofibers}

The surface morphology and internal microstructure of the fabricated PLA-CS fibrous scaffold are shown in Figure 1A. The thin PLA-CS fibers formed a porous interconnected structure and these fibers were randomly oriented in the fibrous scaffold. The structure of the blended PLA-CS nanofiber was similar to that of the natural ECM. The material wettability of the PLA-CS nanofiber was assayed, and the measured contact angle of the PLA-CS nanofiber was $121.17^{\circ}$. This result indicated that the PLA-CS nanofiber is more hydrophobic than a standard culture dish (Su et al., 2010). This characteristic would reduce the interaction between the PLA-CS nanofibers and HepG2 cells, and further promote cellular aggregation to facilitate the formation of multicellular spheroids. The FTIR spectra analysis of the PLA-CS nanofibers exhibited specific peaks for the functional groups from the PLA and CS composition (Figure 1B). Specifically, the peak at $1680 \mathrm{~cm}^{-1}$ is amide I, that at $1540 \mathrm{~cm}^{-1}$ is amide II, that at 1324 $\mathrm{cm}^{-1}$ is amide III for the composition of CS, and the peaks at $1759,1185,1130$, and $1089 \mathrm{~cm}^{-1}$ are the ester groups of PLA. These results revealed that PLA and CS blended well in the PLACS nanofibers.
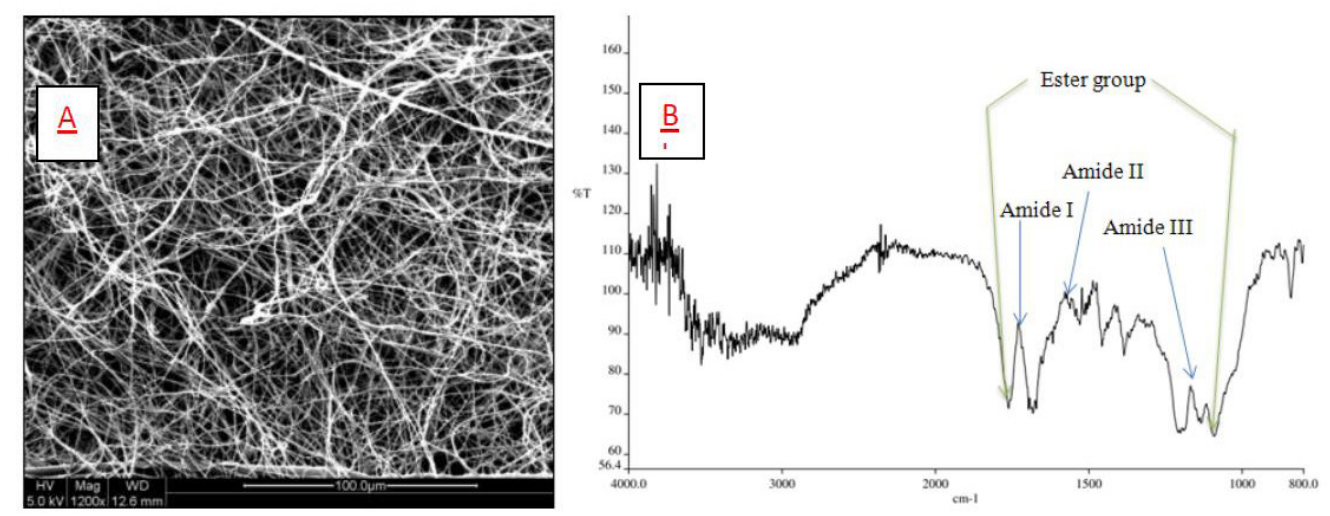

Figure 1. Morphology (A) and FTIR analysis (B) of PLA-CS nanofibers.

\section{Cellular proliferation and aggregation of HepG2 cells}

The viability of HepG2 cells cultured on the PLA-CS fibrous scaffolds was measured

Genetics and Molecular Research 15 (3): gmr.15038993 
using the MTT reagent. MTT analysis is a colorimetric method to examine cellular survival and measure the number of viable cells present. As shown in Figure 2, the PLA-CS nanofiber was found to be suitable for cellular attachment, growth, and viability of HepG2 cells, with only a slight difference compared to growth on 24-well plates. This result is consistent with previous studies of the cellular proliferation on nanofibers (Feng et al., 2010; Su et al., 2015). Following the cultivation period, cellular proliferation increased steadily, and HepG2 cells started to aggregate and formed some cellular clusters such as sphere-like structures. After 4 days of culture, SEM observation revealed that the HepG2 cells formed multicellular spheroids of approximately 100-200 mm diameter (Figure 3A). Figure 3B shows an image of fluorescence immunostaining, revealing multicellular spheroids similar to those observed with SEM. Figure $3 \mathrm{C}$ indicates that that the HepG2 cells could grow into the interior of fibers that could support a 3D-culture space as a natural environment in vivo. The cell assembly aggregated into a spheroid-like multicellular cluster, which could respond to real tissues in the body better than a monolayer of cells. This structure shows promise for several potential applications, including in regenerative medicine and drug development (Lin and Chang, 2008; Lu and Meng, 2012).

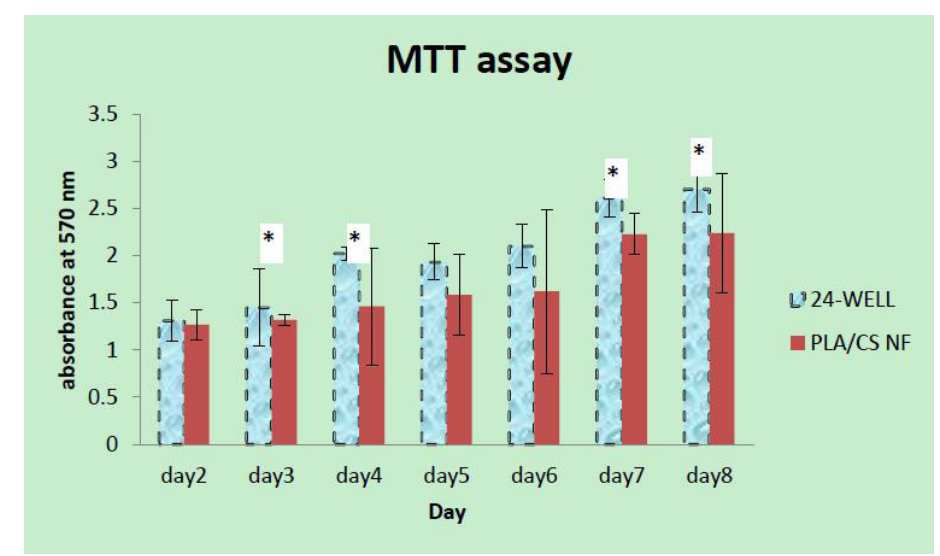

Figure 2. Viability of HepG2 cells seeded on 24-well plates and PLA-CS nanofibers. Cellular proliferation increased steadily. Experiments were performed in triplicate and the values are reported as means $\pm \mathrm{SD}$. Statistical comparisons were conducted; $* \mathrm{P}<0.05$ was considered significant.

A

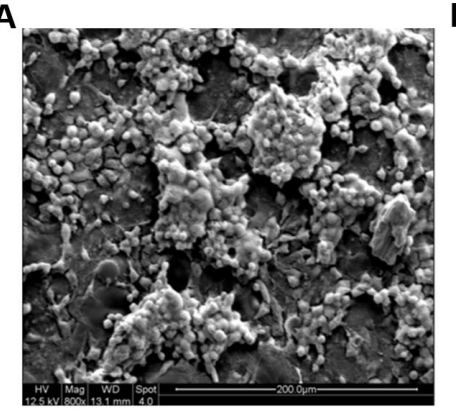

B

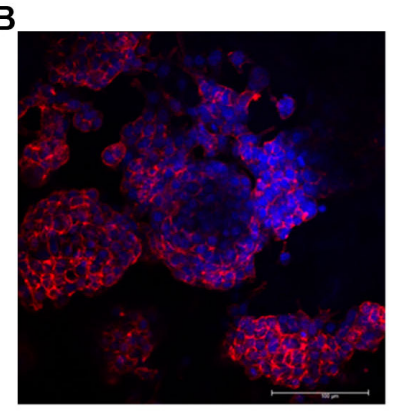

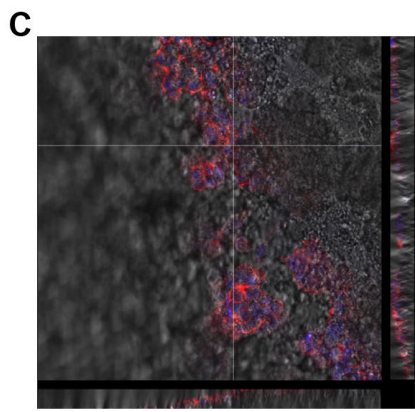

Figure 3. SEM image (A) and image of fluorescence immunostaining; scale bar is $100 \mathrm{~mm}$. (B) Cellular morphology of induced HepG2 cells after 5 days of culture. Cells grew well into the interior of the nanofibers $(\mathbf{C})$. The nucleus is stained blue and red indicates F-actin. 


\section{Liver function analysis}

The secretion of albumin and the synthesis of urea from hepatocytes are useful markers of typical liver function (Jain et al., 2014). The albumin secretion activity of the HepG2 cells cultivated on the PLA-CS nanofibers and 24-well plates at different periods is shown in Figure 4A. HepG2 cells grown on the PLA-CS fibrous scaffold showed dramatically higher levels of albumin secretion than those cultured on the 24-well plates over 7 days, indicating improved hepatic function due to the cellular aggregation to multicellular spheroids on the PLA-CS nanofibers. As shown in Figure 4B, there was also a large difference in urea synthesis of HepG2 cells grown on the PLA-CS nanofibers and 24-well plates, with remarkably greater urea synthesis from cells cultured on the nanofibers. These results indicate that more HepG2 spheroids on the PLA-CS nanofibers can express higher liver activity. In vivo, the majority of cells adhere on ECMs with a nanometer range, indicating that the PLA-CS nanofiber can effectively mimic the topography of ECMs and thereby promote the functional maintenance of primary hepatocytes in vitro (Chu et al., 2009; $\mathrm{Hu}$ and Li, 2015).

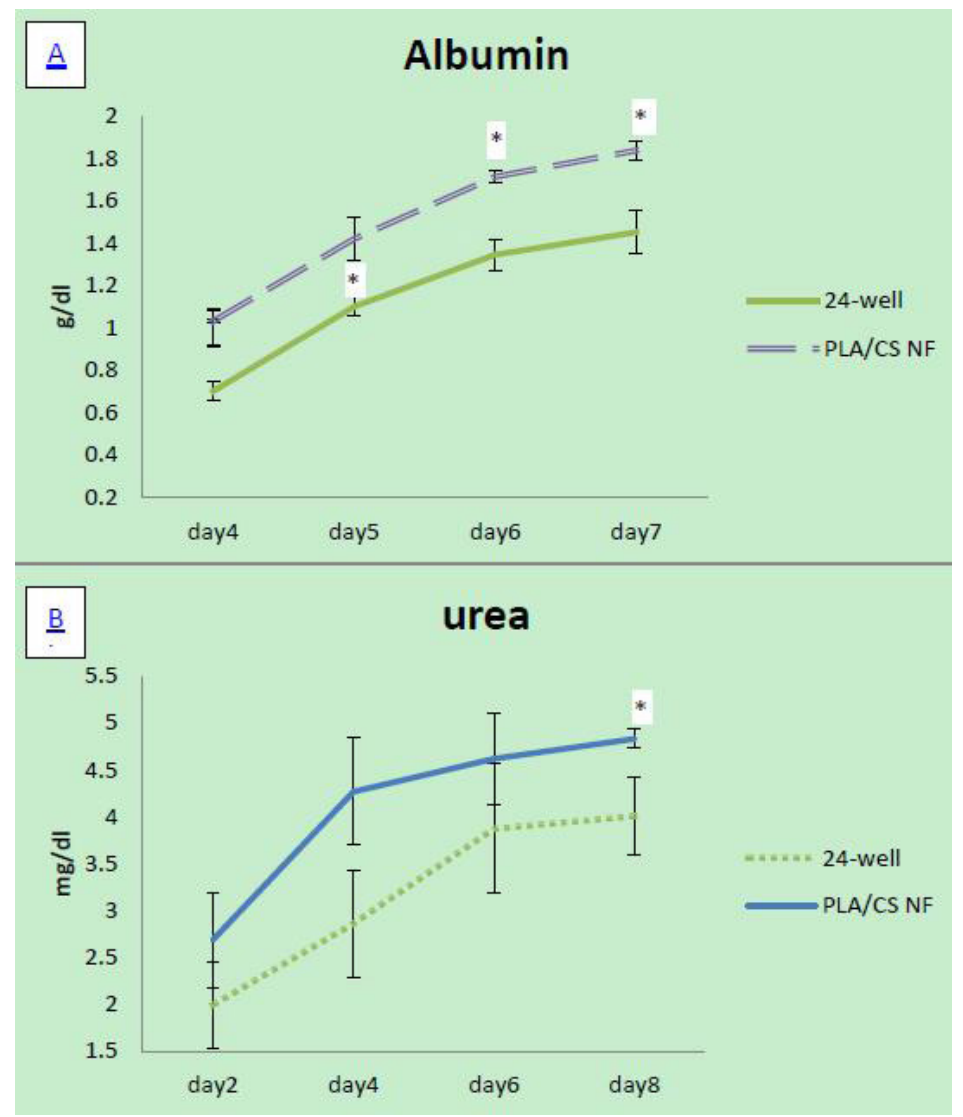

Figure 4. Time courses of albumin secretion (A) and urea synthesis (B) as markers of liver activity from HepG2 spheroids. Experiments were performed in triplicate and the values are reported as means \pm SEM. Statistical comparisons were conducted; $* \mathrm{P}<0.05$ was considered significant.

Genetics and Molecular Research 15 (3): gmr.15038993 


\section{CONCLUSIONS}

This study confirmed that PLA-CS nanofibers are suitable for the growth of HepG2 cells, and can effectively induce formation of an aggregate of HepG2 cells into multicellular spheroids, which further enhances liver function in vitro. This result is supported by the noteworthy higher expression of albumin secretion and urea synthesis from the HepG2 multicellular spheroids. Therefore, PLA-CS nanofibers can be applied to the development of a bioartificial liver to support temporary liver function in vitro. Further studies are needed to achieve a better understanding of the function and properties of various biomaterials for improved design in tissue engineering.

\section{ACKNOWLEDGMENTS}

The authors would like to thank the NTUT-MMH Joint Research Program for financial support (grant \#NTUT-MMH-105-03).

\section{REFERENCES}

Chu XH, Shi XL, Feng ZQ, Gu ZZ, et al. (2009). Chitosan nanofiber scaffold enhances hepatocyte adhesion and function. Biotechnol. Lett. 31: 347-352. http://dx.doi.org/10.1007/s10529-008-9892-1

Feng ZQ, Chu XH, Huang NP, Leach MK, et al. (2010). Rat hepatocyte aggregate formation on discrete aligned nanofibers of type-I collagen-coated poly(L-lactic acid). Biomaterials 31: 3604-3612. http://dx.doi.org/10.1016/j. biomaterials.2010.01.080

$\mathrm{Hu} \mathrm{C}$ and Li L (2015). In vitro culture of isolated primary hepatocytes and stem cell-derived hepatocyte-like cells for liver regeneration. Protein Cell 6: 562-574. http://dx.doi.org/10.1007/s13238-015-0180-2

Jain E, Damania A and Kumar A (2014). Biomaterials for liver tissue engineering. Hepatol. Int. 8: 185-197. http://dx.doi. org/10.1007/s12072-013-9503-7

Lin RZ and Chang HY (2008). Recent advances in three-dimensional multicellular spheroid culture for biomedical research. Biotechnol. J. 3: 1172-1184. http://dx.doi.org/10.1002/biot.200700228

$\mathrm{Lu} \mathrm{Y}$ and Meng Q (2012). Regulation of spheroid formation and function by microenvironmental geometric configuration. Biotechnol. Lett. 34: 37-43. http://dx.doi.org/10.1007/s10529-011-0753-y

Nakazawa K, Lee SW, Fukuda J, Yang DH, et al. (2006). Hepatocyte spheroid formation on a titanium dioxide gel surface and hepatocyte long-term culture. J. Mater. Sci. Mater. Med. 17: 359-364. http://dx.doi.org/10.1007/s10856-006$\underline{8237-7}$

Seo SJ, Kim IY, Choi YJ, Akaike T, et al. (2006). Enhanced liver functions of hepatocytes cocultured with NIH $3 \mathrm{~T} 3$ in the alginate/galactosylated chitosan scaffold. Biomaterials 27: 1487-1495. http://dx.doi.org/10.1016/j. biomaterials.2005.09.018

Su WT, Liao YF, Lin CY and Li LT (2010). Micro-pillar substrate influences the cellular attachment and laminin expression. J. Biomed. Mater. Res. 93A: 1463-1469.

Su WT, Wu PS and Huang TY (2015). Osteogenic differentiation of stem cells from human exfoliated deciduous teeth on poly(E-caprolactone) nanofibers containing strontium phosphate. Mater. Sci. Eng. C 46: 427-434. http://dx.doi. org/10.1016/j.msec.2014.10.076

Genetics and Molecular Research 15 (3): gmr.15038993 